\title{
Photometric observations of weak-line T Tauri stars
}

\author{
II. $w$ tts in Taurus-Auriga, Orion and Scorpius OB2-2 $2^{\star, \star \star}$ \\ C. Chavarría-K ${ }^{1}$, L. Terranegra ${ }^{2}$, M.A. Moreno-Corral ${ }^{1}$, and E. de Lara $^{1}$ \\ 1 Instituto de Astronomía, Carretera Tijuana Ensenada km 107, Ensenada B.C., CP. 22860, México \\ 2 Osservatorio Astronomico di Capodimonte, Via Moiariello 16, I-80131 Napoli, Italy
}

Received March 25, 1999; accepted April 11, 2000

\begin{abstract}
We present uvby- $\beta$ photometry of $116 \mathrm{X}$-ray flux-selected active stars in the directions of the Orion (40), Taurus-Auriga (58) and Scorpius OB2-2 (18) star forming regions. Additionally, we give near IR JHK photometry of 20 active stars in the Taurus-Auriga direction. The program stars were selected from the ROSAT All Sky Survey and EINSTEIN X-ray surveys and are spectroscopically confirmed weak-line $\mathrm{T}$ Tauri stars and weakline $\mathrm{T}$ Tauri star candidates. The photometry confirms the young nature of the program stars and also indicates that a significant fraction of the sample could be foreground objects. The data given here probably represent the largest homogeneous $u v b y-\beta$ photometric sample of new WTTS and WTTS candidates. Many objects in the sample are observed photometrically for the first time.
\end{abstract}

Key words: stars: activity — stars: evolution — stars: pre-main sequence

\section{Introduction}

Soft X-ray surveys of star forming regions (SFRs), for example of Chamaeleon, Lupus, Taurus and Orion, have revealed groups of low-mass $\left(M_{*} \leq 3 M_{\odot}\right)$ active stars apparently associated with those regions (e.g. Alcalá et al. 1995, 1996; Wichmann et al. 1996; Krautter et al. 1997). These objects have late spectral types (typically G0 or later) and are pre-main sequence (PMS) or young (age $\leq$ $10^{8} \mathrm{yr}$ ) main sequence (MS) stars. They are now known as

Send offprint requests to: e-mail: chavarri@astrosen.unam.mx

* Based on observations collected at the Observatorio Astronómico Nacional in Sierra San Pedro Mártir, Baja California, México.

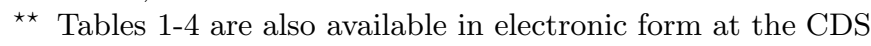
via anonymous ftp to cdsarc.u-strasbg.fr (130.79.128.5) or via http://cdsweb.u-strasbg.fr/Abstract.html weak-line T Tauri stars (WTTS). WTTS are apparently associated with SFRs and, assuming that their distances to the observer are those of their associated regions, one infers their youth from their positions in the $\mathrm{H}-\mathrm{R}$ diagram. Besides these morphological and photometric properties, the WTTS also present key features in their spectra that reveal us their young (age $<10^{8}$ ) nature: they have chromospheric activity $(\mathrm{H} \alpha$ and $\mathrm{CaII} \mathrm{H}+\mathrm{K}$ lines in emission, $W(\mathrm{H} \alpha) \leq-10 \AA$ ), the photospheric line LiI $\lambda 6708 \AA$ is conspicuously present in their spectra) and the stars rotate fast $\left(v_{\text {rot }} \approx 30 \mathrm{~km} \mathrm{~s}^{-1}\right.$ or more). A necessary condition to classify these objects as PMS stars is the presence of the $\mathrm{Li} I$ feature in excess in the $W(\mathrm{Li})$ versus $\log T_{\text {eff }}$ plane when compared with the upper envelope of the Pleiades stars (e.g. Fig. 12 of Alcalá et al. 1998, hereafter A98). In this case, Lithium has not had enough time to be destroyed in the deeper layers of the convection zone (cf. Bodenheimer 1965). On the other hand, the WTTS are mildly masked, if at all, by circumstellar material: they lack (strong) spectral line and continuum (ultraviolet or infrared) emissions that characterize the classical T Tauri stars (CTTS), which are believed to be younger (age $\leq 10^{7} \mathrm{yr}$ ). WTTS are considered the descendants of $C T T S$. However, the criteria for establishing the membership of the WTTS to an ongoing star forming region should be taken with caution because of their large spatial distribution $\left(>100 \square^{0}\right)$ and because their distance to the observer is, in most cases, unknown.

Although the EINSTEIN satellite shed new light on the evolution of these PMS objects, its observations were spatially biased towards the denser parts of the SFRs, where CTTS predominate and follow a clumpy pattern. Hence, little or no inference could be drawn about the history of star formation at a cloud scale. The spatially unbiased ROSAT All-Sky Survey (RASS) remedies this situation: it yields a spatially complete but fluxlimited sample of X-ray sources around a given cloud at about EINSTEIN's sensitivity. Contrary to the spatial 
distribution of the CTTS, WTTS were found to be uniformly distributed over the whole observed area and outnumber the preceding $C T T S$ by, at least, a factor of 3 . The location of the WTTS in the evolutionary sequence has not yet been well established (e.g. Montmerle et al. 1993; Chavarría-K et al. 1995), but in general, stellar activity is considered a quantitative landmark of youth for this type of objects. Since WTTS are less active in the $u v$, optical and infrared spectral regions than $C T T S$, the former are considered older than the latter. Consequently, WTTS had more time to disperse from their birth sites, explaining, at least partially, their broader and homogeneous spatial distribution. Despite the intrinsic X-ray brightness of the WTTS, we are luminosity-limited in their detection. At present, we are constrained to only nearby SFRs $(d<500 \mathrm{pc})$.

Many follow-up studies (optical, mostly spectroscopic) of the EINSTEIN and ROSAT surveys of nearby SFRs such as Chameleon, Taurus-Auriga, Lupus, Orion and Scorpius-Centaurus have recently appeared in the literature (e.g. Alcalá et al. 1995; Wichmann et al. 1996, 1997; Li \& Hu 1998; Magazzù et al. 1997; Krautter et al. 1997; Alcalá et al. 1996, 1998; Walter et al. 1988, 1994; Sciortino et al. 1998 and references therein). Unfortunately, photometric data of many WTTS and WTTS candidates associated with the regions of interest are lacking or the stars were observed in an unsuitable photometric system (e.g. the photographically-based GSC or satellite-born $V$ magnitude estimates, particularly for red stars): the exceptions are the monitoring for light-variability of 58 WTTS (and WTTS candidates) in Taurus by Bouvier et al. (1997) in Johnson's $B$ and $V$ passbands, the $u v b y-\beta$ photometry of WTTS and WTTS candidates in Orion by Alcalá et al. (1996, hereafter A96) and the UBVRIJHKL photometry of WTTS in Scorpius OB2-2 by Walter et al. (1994, hereafter W94).

In this paper we report and discuss photometric data of X-ray flux selected and confirmed WTTS and WTTS candidates in the Taurus-Auriga SFR (uvby- $\beta$ and $J H K, 58$ and 20 stars, respectively) and in Scorpius OB2-2 SFR (uvby- $\beta, 18$ stars). We also extend the photometric sample of WTTS in Orion reported by A96 (uvby- $\beta, 40$ stars). The majority of stars are observed for the first time in this photometric system. The data cover about $74 \%$ of the objects on the list by Wichmann et al. (1996, hereafter Wi96), about $36 \%$ on the list by A96, and about $46 \%$ from the list of WTTS and WTTS candidates in the Upper Scorpius Association by Wa94, as well as six objects near the runaway O star $\zeta$ Oph (Oph1 through Oph6, Walter 1986, see also Terranegra et al. 1994).

In spite of previous spectroscopic, photometric and proper motion studies, the membership of WTTS to clusters with ongoing star formation is still an open matter. It is the purpose of this work to cover some of these deficiencies for future analysis and observations.

\section{Observations and data analysis}

The program stars were taken from the spectroscopic follow-up surveys of ROSAT X-ray sources in Orion and Taurus-Auriga SFRs by A96 and Wi96, respectively, and from a study of EINSTEIN X-ray sources in Scorpius OB2-2 SFR by Wa94 and of X-ray sources east of the Scorpius-Centaurus-Lupus OB association (Walter 1986, hereafter Wa86). Our uvby- $\beta$ observations complement these previous studies. They avoid systematic errors due to different observers, equipment, calibrations or reduction procedures and represent a homogeneous large photometric sample of WTTS and WTTS candidates.

For convenience purposes, the spatial distributions of our program stars in Taurus-Auriga and Scorpius OB2-2 association are shown in Figs. 1 and 2, respectively.

\subsection{The near IR photometry of Taurus-Auriga stars}

The JHK photometric data of 20 active stars in TaurusAuriga as presented in Table 1 were collected with the $2.1 \mathrm{~m}$ telescope of the Observatorio Astronómico Nacional in Sierra San Pedro Mártir (SPMO, Baja California (México) in November/December 1992. The telescope was used in the $\mathrm{f} / 27 \mathrm{IR}$ configuration mode with a N2-cooled InSb diode detector. The chopping frequency was set to $10 \mathrm{~Hz}$ and a throw of $30^{\prime \prime}$ in the delta direction and a $14^{\prime \prime}$ diaphragm were used throughout the observing run. The set of standard stars for San Pedro Mártir Observatory given by Tapia et al. (1986) was used here to tie the observations to the standard system (see also Carrasco et al. 1991). At least four standard stars were observed nightly. The photometry was reduced following usual procedures (e.g. Mitchell 1960; Chavarría et al. 2000). Details on the equipment, observing procedures and quality of the resulting photometry are given in A96 (see also references therein and remarks in A98), since the equipment and set of standards used are the same in both works. The standard errors of the IR magnitudes of Table 1 are typically $\sigma_{J}=0.03, \sigma_{H}=0.04$ and $\sigma_{K}=0.04$.

\subsection{The uvby- $\beta$ photometry of program stars}

The $u v b y-\beta$ photometry of 116 active PMS stars associated with Orion, Taurus-Auriga and Scorpius OB2-2 SFRs was collected during four observing runs from 1994 to 1996 at SPMO using the Danish six channel grating spectrophotometer attached to the Harold L. Johnson $1.5 \mathrm{~m}$ telescope. The photometer consists of an entrance slit, a grating as its dispersing element, six exit slots and filters that match the Strömgren/Crawford original filters fairly well and six uncooled photomultipliers as light detectors in the photon counting mode. Four channels are used for simultaneous uvby observations and two for the $\beta$-index measurements (the "wide" and "narrow" $\mathrm{H} \beta$ passbands, which 


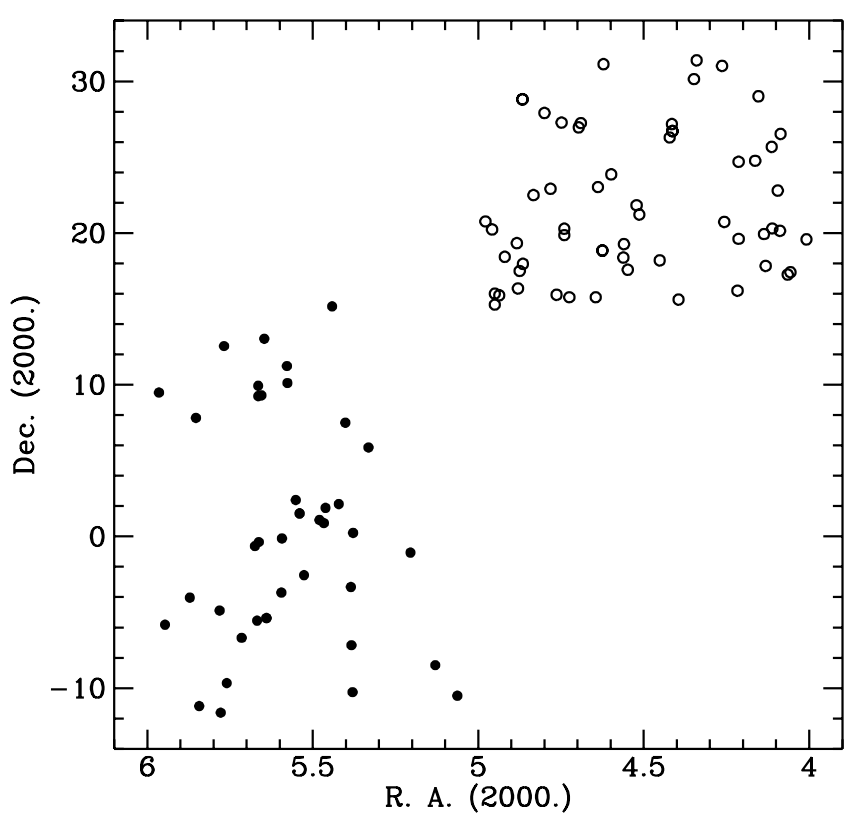

Fig. 1. The spatial distribution of the RASS WTTS in TaurusAuriga (open circle) and in Orion SFRs (filled circle)

are measured simultaneously). One switches swiftly from one observing mode to the other with a computer command from the data acquisition system. When observing any given star, we made the $\beta$-measurements immediately after the uvby observations. For more details about the instrument see Nissen (1984); Terranegra et al. (1994, T94 hereinafter) and A96. The integration time on each star was normally fixed in order to achieve a photon noise of less than $1 \%$ in the weakest flux of a given passband (usually $u$ or $v$ ). Sky was normally measured $30^{\prime \prime}$ east of the star and, if necessary, intercalated between two 30 second integrations of the star + sky measurements as many times as necessary, until the photon noise error of $1 \%$ could be achieved. Some program stars could not be measured with the quoted accuracy. The $\beta$ index was usually determined with a $2 \%$ or better precision. Finally, the seasonal standard stars were each observed twice or more in, at least, one of the four independent runs. The data were reduced to the standard system following usual procedures (e.g. A96, T94, Chavarría et al. 2000). The standard system is taken from the lists by Crawford et al. (1971, 1973) and by Olsen $(1983,1984)$. On average, about 85 standard stars were observed per season and constitute over 100 standard stars which were used to tie the observations of the different runs to the $u v b y-\beta$ standard system. We took care to include reference stars covering the spectral types (F5-M5) and luminosity classes (V and III) of the program stars. The resulting uvby- $\beta$ photometry of the program stars observed here is displayed in Tables 2 to 4 . The typical standard errors for the photometry of the program stars with $V \leq 13^{\mathrm{m}}$ are $\sigma_{V}=0.010, \sigma_{b-y}=0.013$, $\sigma_{m 1}=0.015, \sigma_{c 1}=0.020$ and $\sigma_{\beta}=0.02$ and thrice as large for stars with $V \geq 14^{\mathrm{m}}$.

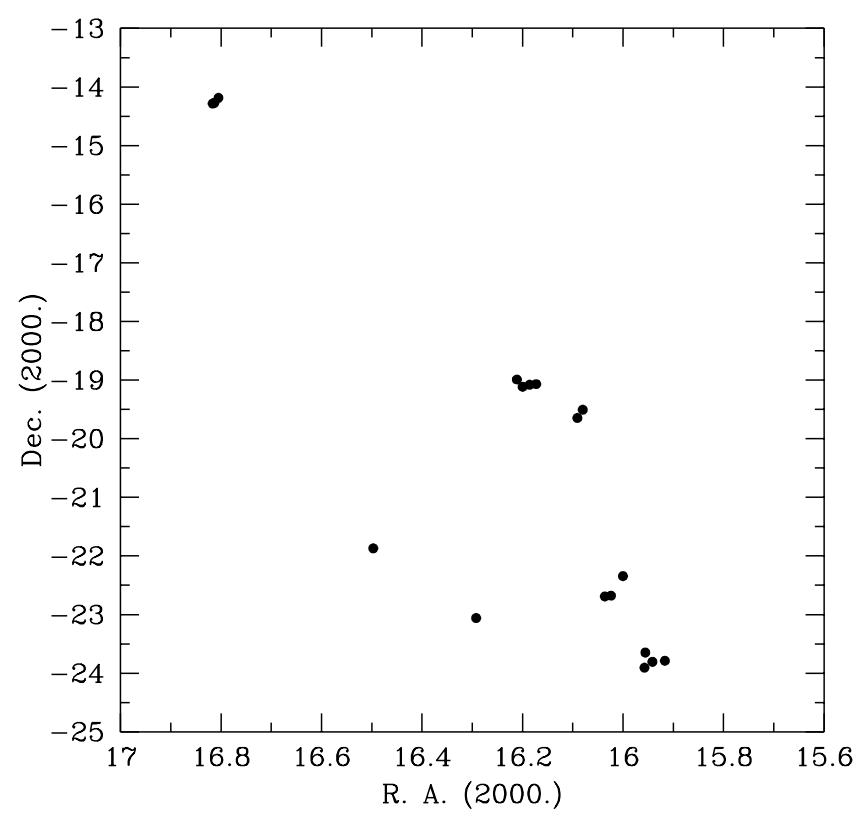

Fig. 2. The spatial distribution of the EO WTTS in Scorpius OB2-2

\section{Results and discussion}

\subsection{Apparent magnitude distributions}

Using the data of Tables 2 to 4 we have compared the apparent $V$ magnitude distributions (VD) of WTTS with their CTTS counterparts in Taurus-Auriga, Orion and Scorpius OB2-2 SFRs. The results are shown in Fig. 3, where the data for the CTTS were taken from the Herbig and Bell Catalog (1988) in order to fit the range of spectral types observed: G-M in Orion and K-M in Taurus-Auriga and Scorpius OB2-2. Note that there are no CTTS with spectral types earlier than K0 in Taurus-Auriga (cf. Herbig \& Bell 1988). The $V$-distribution of Orion's WTTS given by us here (cf. Fig. 3a, hatched area) differs from that reported by A98 (their Fig. 2) because our sample covers the weaker end of the stars better. The $V$-distribution of the WTTS in Taurus-Auriga is shown in Fig. 3b (hatched area), together with the distribution of the WTTS in Scorpius OB2-2 (shaded area) but scaled to the distance of Taurus-Auriga. This was done because of the scarcity of CTTS reported in Scorpius OB2-2 (cf. Herbig \& Bell 1988).

Again, as in the case of Orion reported by A98, we see that there is a significant number of WTTS brighter than the mean brightness of CTTS, i.e. the bona fide members of the SFRs, indicating us that some of the program stars could be foreground stars. On the other hand, if the objects do belong to the SFR, then many $W T T S$ will be more massive and younger than their predecessors, the $C T T S$, in contradiction with the evolutionary scheme for pre-main sequence stars. Many stars would be only a few million years old and, hence, have not had 
Table 1. JHK photometry of new WTTS in Taurus-Auriga

\begin{tabular}{lrrr}
\hline RXJ & $J$ & \multicolumn{1}{c}{$H$} & \multicolumn{1}{c}{$K$} \\
\hline & & & \\
$0409.3+1716$ & 11.84 & 11.57 & 11.49 \\
$0412.8+1937$ & 9.88 & 9.19 & 9.04 \\
$0420.3+3123$ & 10.32 & 9.74 & 9.63 \\
$0425.3+2618^{1}$ & 9.92 & 8.62 & 8.10 \\
$0433.7+1823$ & 9.99 & 9.37 & 9.27 \\
$0433.9+2613^{2}$ & 9.65 & 8.37 & 7.77 \\
$0437.3+3108$ & 10.47 & 9.58 & 9.44 \\
$0437.5+1851 \mathrm{~A}+\mathrm{B}$ & 9.12 & 8.24 & 8.10 \\
$0441.4+2715$ & 11.05 & 10.51 & 10.45 \\
$0443.5+1546$ & 10.76 & 10.11 & 10.02 \\
$0444.4+2017$ & 10.30 & 9.59 & 9.47 \\
$0444.4+1952$ & 9.60 & 8.75 & 8.61 \\
$0444.9+2717^{3}$ & 7.74 & 7.22 & 7.14 \\
$0451.9+1758$ & 10.23 & 9.46 & 9.27 \\
$0452.0+2849 \mathrm{~A}+\mathrm{B}$ & 10.70 & 10.06 & 9.84 \\
$0452.5+1730$ & 9.46 & 8.43 & 8.24 \\
$0453.0+1920$ & 10.01 & 9.36 & 9.22 \\
$0456.6+3150^{4}$ & 7.82 & 7.16 & 7.04 \\
$0457.5+2014$ & 9.25 & 8.73 & 8.59 \\
$0458.7+2046$ & 9.55 & 8.89 & 9.77 \\
& & & \\
\hline
\end{tabular}

Remarks to table:

${ }^{1}$ J4872; ${ }^{2}$ IT Tau; ${ }^{3} \mathrm{HD} 283782{ }^{4} \mathrm{HD} 282598$.

the time to disperse. Moreover, one should explain why several WTTS get rid of the circumstellar envelope at a faster rate than their predecessors, the CTTS. From Fig. $3 \mathrm{~b}$ we also note that a large fraction of WTTS is brighter than the mean brightness of their CTTS counterparts. This is more evident if we also consider the large fraction of WTTS in Taurus-Auriga with spectral types earlier than K0 (16 stars). One would expect from the stellar evolution theory that the younger stars, ie. the CTTS, are more luminous than the older WTTS (e.g. Shu et al. 1987), in contrast to what we observe in Fig. 3.

The apparent greater brightness of the WTTS relative to their CTTS counterparts (cf. Fig. 3) could be due to at least three causes: i) CTTS are intrinsically fainter than the WTTS, ii) WTTS are affected by less IS extinction and circumstellar extinction then their CTTS counterparts, and iii) WTTS are effectively nearer to the Sun than the CTTS. In the first case, from a direct comparison between the spectral class distributions of the two types of stars we see a two subclass shift between their respective maxima. This small shift in their spectral types accounts for only about 0.5 of the almost $2^{\mathrm{m}}$ magnitude difference shown in their respective VDs of Fig. 3, minimizing this possibility. With respect to the second case, we do expect, on the average, CTTS to be more reddened than their WTTS counterparts since the former are masked by circumstellar matter and the latter not. Using the photometry of Tables 2 to 4 , the spectral types
Table 2. uvby- $\beta$ photometry of the WTTS in Orion

\begin{tabular}{|c|c|c|c|c|c|c|c|}
\hline$\overline{\mathrm{RXJ}}$ & Sp.T. & $\bar{V}$ & $b-y$ & $m 1$ & $c 1$ & $\bar{\beta}$ & $A_{V}$ \\
\hline $0503.8-1130$ & K3 & 12.26 & 0.49 & 0.28 & 0.23 & 2.59 & 0.00 \\
\hline $0507.8-0931$ & $\mathrm{~K} 2$ & 12.50 & 0.55 & 0.27 & 0.31 & 2.59 & 0.11 \\
\hline $0519.9+0552$ & K6 & 14.72 & 0.78 & 0.58 & 0.37 & 2.59 & 0.36 \\
\hline $0522.7+0014 \dagger$ & M2 & 15.73 & 1.02 & 0.63 & -0.46 & 2.43 & 0.83 \\
\hline $0522.8-1144 \dagger$ & M1 & 14.74 & 0.97 & 0.31 & -0.30 & 2.44 & 0.68 \\
\hline $0523.0-0850$ & K7M0 & 14.60 & 0.97 & 0.41 & 0.32 & 2.54 & 1.02 \\
\hline $0523.1-0440$ & K5 & 14.68 & 0.66 & 0.58 & -0.05 & 2.58 & 0.00 \\
\hline $0524.1+0730$ & $\mathrm{~K} 4$ & 12.72 & 0.60 & 0.31 & 0.37 & 2.59 & 0.00 \\
\hline $0525.3+0208$ & M4 & 15.77 & 1.00 & 0.27 & 0.34 & 2.42 & 0.47 \\
\hline $0526.5+1510$ & G5 & 11.85 & 0.43 & 0.20 & 0.37 & 2.61 & 0.15 \\
\hline $0527.7+0153 \dagger$ & $\mathrm{K} 7$ & 16.02 & 0.76 & 1.00 & -0.21 & 2.69 & 0.00 \\
\hline $0528.0-0053$ & K0 & 12.72 & 0.53 & 0.37 & 0.25 & 2.59 & 0.31 \\
\hline $0528.8+0105$ & K4 & 12.67 & 0.65 & 0.59 & 0.27 & 2.56 & 0.23 \\
\hline $0531.6-0327$ & K0 & 9.54 & 0.56 & 0.37 & 0.28 & 2.58 & 0.46 \\
\hline $0532.4+0131 \mathrm{a}$ & $\mathrm{K} 2$ & 11.98 & 0.51 & 0.22 & 0.35 & 2.60 & 0.00 \\
\hline $0532.4+0131 b$ & K5 & 13.78 & 0.70 & 0.42 & 0.30 & 2.58 & 0.20 \\
\hline $0533.1+0224$ & $\mathrm{~K} 4$ & 13.49 & 0.65 & 0.38 & 0.23 & 2.56 & 0.23 \\
\hline $0534.6+1007$ & K3 & 9.91 & 0.54 & 0.23 & 0.29 & 2.58 & 0.00 \\
\hline $0534.7+1114$ & $\mathrm{~K} 4$ & 12.41 & 0.51 & 0.26 & 0.28 & 2.58 & 0.00 \\
\hline $0535.6-0152$ & G9 & 11.90 & 0.45 & 0.21 & 0.33 & 2.61 & 0.00 \\
\hline $0535.7-0418 \dagger$ & K3 & 14.56 & 0.63 & 0.81 & -0.24 & 2.49 & 0.40 \\
\hline $0538.4-0637 \mathrm{a}$ & $\mathrm{K} 1$ & 12.28 & 0.63 & 0.40 & 0.30 & 2.58 & 0.71 \\
\hline $0538.4-0637 b$ & $\mathrm{~K} 2$ & 12.99 & 0.58 & 0.36 & 0.21 & 2.55 & 0.27 \\
\hline $0538.8+1302$ & $\mathrm{~K} 2$ & 10.95 & 0.44 & 0.19 & 0.32 & 2.60 & 0.00 \\
\hline $0539.3+0918$ & K1 & 11.71 & 0.52 & 0.35 & 0.28 & 2.59 & 0.10 \\
\hline $0539.8-0138$ & K3 & 13.01 & 0.67 & 0.54 & 0.17 & 2.58 & 0.62 \\
\hline $0539.9+0915$ & K0 & 11.50 & 0.53 & 0.32 & 0.31 & 2.58 & 0.31 \\
\hline $0539.9+0956$ & K4 & 10.91 & 0.51 & 0.33 & 0.27 & 2.58 & 0.00 \\
\hline $0540.1-0627 \dagger$ & K7M0 & 15.11 & 0.78 & 0.11 & -0.14 & 2.46 & 0.00 \\
\hline $0540.5-0122$ & K5 & 10.40 & 0.33 & 0.12 & 0.45 & 2.65 & 0.00 \\
\hline $0542.9-0719 \dagger$ & M3 & 14.39 & 1.07 & 0.67 & 0.70 & 2.47 & 1.01 \\
\hline $0545.6-1020$ & G7 & 13.61 & 1.23 & 0.22 & 1.5: & 2.59 & 4.43 \\
\hline $0546.1+1233$ & G9 & 11.76 & 0.44 & 0.18 & 0.28 & 2.57 & 0.00 \\
\hline $0546.7-1223$ & G5 & 13.30 & 0.41 & 0.29 & 0.34 & 2.57 & 0.04 \\
\hline $0546.9-0507 \dagger$ & K4 & 12.30 & 0.85 & 0.34 & 0.26 & 2.59 & 1.35 \\
\hline $0550.6-1249 \dagger$ & K6 & 13.43 & 0.71 & 0.45 & 0.78 & 2.39 & 0.00 \\
\hline $0551.2+0749 \dagger$ & K6 & 12.35 & 0.57 & 0.49 & -0.02 & 2.51 & 0.00 \\
\hline $0552.3-0558$ & $\mathrm{~K} 2$ & 13.09 & 0.48 & 0.31 & 0.16 & 2.70 & 0.00 \\
\hline $0556.8-0611 \dagger$ & K5 & 11.84 & 0.70 & 0.42 & 0.19 & 2.64 & 0.20 \\
\hline $0557.9+0929$ & G9 & 11.35 & 0.42 & 0.17 & 0.32 & 2.60 & 0.00 \\
\hline
\end{tabular}

Notes to Table:

$\dagger$ After A96, the presence of LiI $6707 \AA$ dubious because of the low S/N ratio of the spectrogram.

reported for the program stars in the literature (A96, Wi96 and Wa94) and the intrinsic colours given by Olsen (1984) we have computed the visual extinction of our program stars. The resulting values are reported in the last column of Tables 2 to 4 . We find that the 40 program stars in Orion are reddened, on average, $A_{V}=0.36 \pm 0.11$ with a median of 0.13 , while the 58 objects in Taurus-Auriga give $A_{V}=0.74 \pm 0.09$ with a median of 0.49 . Restricting the spectral type ranges of the CTTS given by Cohen \& Kuhi (1979) to coincide with the spectral types of the program star in Taurus-Auriga and Orion SFRs (mainly $\mathrm{K} 0-\mathrm{K} 7 / \mathrm{M} 0)$ and using the $A_{V}$ estimates for the CTTS by Cohen \& Kuhi, we find that, on average, the CTTS are more reddened by $\delta A_{V}=0.37 \pm 0.15$ and $=0.79 \pm 0.31$ in Orion and Taurus-Auriga, respectively. Again, this cannot account for the $2^{\mathrm{m}}$ difference in brightness we observe 

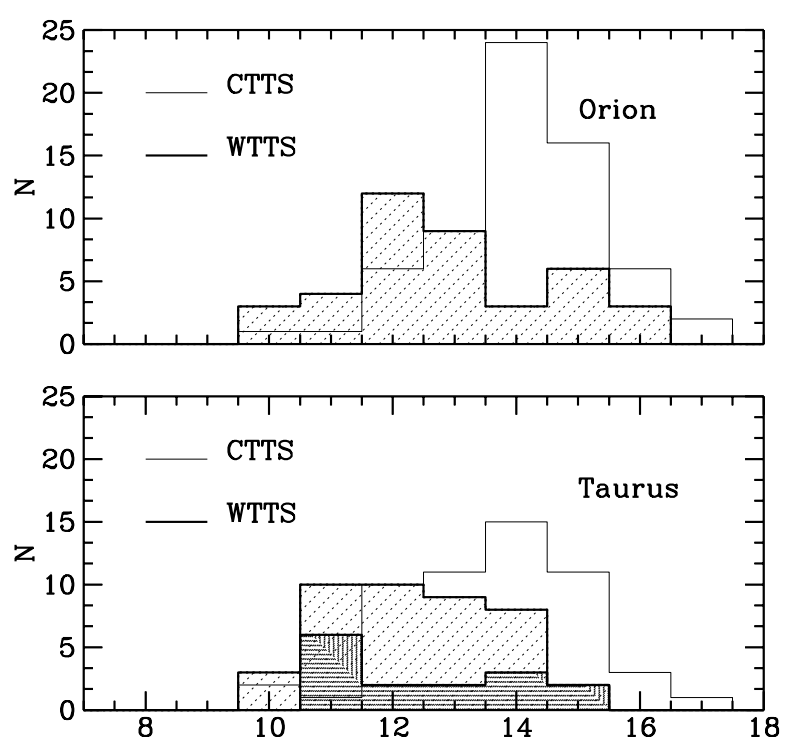

Fig. 3. The apparent $V$ magnitude distributions (VD) of the WTTS studied here. In the upper panel the VD of the program WTTS in Orion (thick line) is compared with that of the CTTS of the same SFR (thin line). In the lower panel we show the VDs of the WTTS and the CTTS in Taurus-Auriga, taken from Herbig \& Bell (1988). For comparison we also include the WTTS in Scorpius OB2-2 scaled to the distance of Taurus-Auriga SFR (shaded area). The samples of CTTS were selected in order to fit the range of spectral types observed in Orion (G-M) and in Taurus-Auriga and Scorpius OB2-2 (K-M)

(cf. Fig. 3). Hence, we conclude that, for a given spectral subclass, at least the upper brightest stars in the case of Taurus-Auriga could be foreground objects, the case of Orion being even more obvious.

\subsection{Magnitude-colour and two-colour diagrams}

In Figs. 4, 5 and 6 we show the observed magnitude $V$ versus colour $(b-y)$ diagrams for the program stars associated with Orion, Taurus-Auriga and Scorpius OB2-2, respectively. Also depicted in the figures are the ZAMS from Crawford (1979) and Olsen (1984) scaled to a distance of $460 \mathrm{pc}, 140 \mathrm{pc}$ and $160 \mathrm{pc}$ for Orion, Taurus-Auriga and Scorpius OB2-2, respectively. The arrows on the figures indicate the reddening vector. From the location of the program stars in their respective colour magnitude diagram and considering the mean $A_{V}$ values for the three SFRs, there are indications that the brightest stars could be foreground objects, as mentioned before, others could be reaching the ZAMS and, since they lay below the main sequence at the distance of a given SFR, a few could be background stars.

In Figs. 7 and 8 we show the location of the program stars in the reddening-free $\left(\left[m_{1}\right],\left[c_{1}\right]\right)$ and the $\left(\beta,\left[m_{1}\right]\right)$ diagrams, respectively (Orion filled circles, Taurus-Auriga open circles, Scorpius OB2-2 diamonds). We also show
Table 3. $u v b y-\beta$ photometry of the WTTS in Taurus-Auriga

\begin{tabular}{|c|c|c|c|c|c|c|c|}
\hline Name/RXJ & Sp.T. & $\bar{V}$ & $b-y$ & $m 1$ & $c 1$ & $\beta$ & $A_{V}$ \\
\hline HD 285281 & K1 & 10.14 & 0.59 & 0.31 & 0.31 & 2.57 & 0.49 \\
\hline $0403.3+1725$ & K3 & 11.69 & 0.66 & 0.54 & 0.12 & 2.52 & 0.72 \\
\hline $0405.1+2632$ & $\mathrm{~K} 2$ & 11.53 & 0.55 & 0.32 & 0.24 & 2.55 & 0.26 \\
\hline $0405.3+2009$ & $\mathrm{~K} 1$ & 10.41 & 0.57 & 0.36 & 0.28 & 2.55 & 0.22 \\
\hline HD 284135 & G3 & 9.37 & 0.43 & 0.12 & 0.36 & 2.60 & 0.31 \\
\hline HD 284149 & G1 & 9.51 & 0.39 & 0.11 & 0.40 & 2.62 & 0.18 \\
\hline $0406.8+2541$ & K7M0 & 11.72 & 0.87 & 0.54 & -0.11 & 2.41 & 1.46 \\
\hline $0407.8+1750$ & $\mathrm{~K} 4$ & 11.27 & 0.52 & 0.24 & 0.17 & 2.53 & 0.10 \\
\hline $0408.2+1956$ & $\mathrm{~K} 2$ & 13.05 & 0.87 & 0.14 & 0.44 & 2.47 & 1.74 \\
\hline $0409.2+2901$ & K1 & 10.64 & 0.53 & 0.31 & 0.26 & 2.55 & 0.53 \\
\hline $0409.3+1716$ & M1 & 13.30 & 0.95 & 0.51 & 0.60 & 2.54 & 0.56 \\
\hline $8+2446$ & M1.5 & 13.30 & 0.95 & 0.37 & 0.27 & 2.54 & 0.50 \\
\hline $0412.8+1937$ & K6 & 12.56 & 0.81 & 0.74 & -0.03 & 2.53 & 0.47 \\
\hline $0412.8+2442$ & G9 & 11.97 & 0.76 & 0.04 & 0.44 & 2.60 & 1.81 \\
\hline HD 285579 & G1 & 10.92 & 0.50 & 0.07 & 0.35 & 2.65 & 0.80 \\
\hline $0415.4+2044$ & K0 & 10.67 & 0.49 & 0.20 & 0.31 & 2.59 & 0.30 \\
\hline $0415.9+3100$ & G6 & 12.36 & 0.60 & 0.18 & 0.30 & 2.62 & 1.16 \\
\hline $0420.3+3123$ & $\mathrm{~K} 4$ & 11.76 & 0.60 & 0.29 & 0.38 & 2.61 & 0.00 \\
\hline $0420.9+3009$ & K7M0 & 14.74 & 0.95 & 0.38 & 0.82 & 2.67 & 0.78 \\
\hline HD 285751 & $\mathrm{~K} 2$ & 11.25 & 0.60 & 0.34 & 0.31 & 2.53 & 0.70 \\
\hline $\mathrm{BD}+26718$ & $\mathrm{~K} 1$ & 11.45 & 0.92 & 0.12 & 0.43 & 2.57 & 2.33 \\
\hline $\mathrm{BD}+26718 \mathrm{~B}$ & K0 & 11.47 & 0.91 & 0.10 & 0.47 & 2.55 & 2.27 \\
\hline $0424.9+2711 \dagger$ & M0.5 & 13.42 & 0.89 & 0.44 & -0.38 & 2.44 & 0.32 \\
\hline $3+2618 \ddagger$ & K7 & 13.60 & 1.08 & 0.12 & 0.23 & 2.29 & 1.76 \\
\hline $\mathrm{BD}+17724 \mathrm{~B}$ & G5 & 9.44 & 0.40 & 0.12 & 0.37 & 2.63 & 0.00 \\
\hline $0430.8+2113$ & G8 & 10.40 & 0.47 & 0.21 & 0.33 & 2.59 & 0.19 \\
\hline 84496 & K0 & 10.96 & 0.52 & 0.29 & 0.33 & 2.59 & 0.25 \\
\hline $0432.8+1735$ & M2 & 13.69 & 1.07 & 0.48 & -0.35 & 2.40 & 1.11 \\
\hline $0433.5+1916$ & G6 & 13.58 & 0.71 & 0.16 & 0.37 & 2.60 & 0.56 \\
\hline $0433.7+1823$ & G6 & 12.05 & 0.70 & 0.06 & 0.42 & 2.62 & 1.66 \\
\hline $0435.9+2352$ & M1.5 & 14.48 & 1.00 & 0.35 & 0.37 & 2.52 & 0.72 \\
\hline $0437.3+3108$ & K4 & 13.80 & 0.91 & 0.25 & 0.41 & 2.73 & 2.34 \\
\hline $0437.4+1851 \mathrm{~A}$ & K6 & 1.84 & 0.69 & 0.70 & -0.03 & 2.61 & 0.00 \\
\hline $351 \mathrm{~B}$ & M0.5 & 3.45 & 0.90 & 0.70 & -0.27 & 2.59 & 0.50 \\
\hline+2302 & M1 & .42 & 0. & & 0.39 & 2.50 & 0.66 \\
\hline 285957 & K1 & 11.05 & 0.54 & 0.43 & 0.28 & 2.57 & 0.05 \\
\hline $0441.4+2715$ & G8 & 13.50 & 0.81 & 0.29 & 0.97 & 2.88 & 2.09 \\
\hline 83798 & G7 & 9.83 & 0.40 & 0.29 & 0.32 & 2.60 & 0.00 \\
\hline $0443.4+1546$ & G7 & 12.93 & 0.72 & 0.14 & 0.61 & 2.65 & 1.59 \\
\hline $0444.3+2017$ & K1 & 12.66 & 0.73 & 0.35 & 0.23 & 2.63 & 1.27 \\
\hline $0444.4+1952$ & M1 & 12.59 & 0.93 & 0.58 & 0.47 & 2.58 & 0.45 \\
\hline $0444.9+2717$ & K1 & 9.71 & 0.64 & 0.25 & 0.31 & 2.61 & 0.77 \\
\hline HD 30171 & G5 & 9.37 & 0.48 & 0.23 & 0.35 & 2.59 & 0.43 \\
\hline $0446.8+2255$ & M1 & 12.94 & 0.85 & 0.66 & -0.07 & 2.58 & 0.00 \\
\hline $9+2755$ & K0 & 12.39 & 0.77 & - & 0.50 & 2.59 & 1.77 \\
\hline $0450.0+2230$ & K1 & 11.08 & 0.55 & 0.30 & 0.27 & - & 0.26 \\
\hline $8+1758$ & M1.5 & 1.26 & 0.98 & 0.94 & 0.49 & 1.95 & 0.73 \\
\hline $0451.9+2849 \mathrm{~A}$ & K4 & 14.38 & 1.00 & -0.01 & 0.76 & 2.57 & 2.46 \\
\hline $0451.9+2849 \mathrm{~B}$ & K2 & 14.52 & 0.76 & 0.14 & 0.52 & 2.51 & 1.28 \\
\hline $0452.5+1730$ & K4 & 12.02 & 0.64 & 0.56 & 0.20 & 2.56 & 0.17 \\
\hline $0452.8+1621$ & K6 & 11.65 & 0.80 & 0.60 & 0.10 & 2.49 & 1.07 \\
\hline $0452.9+1920$ & K5 & 12.15 & 0.66 & 0.51 & -0.01 & 2.61 & 0.00 \\
\hline HD 31281 & G1 & 9.31 & 0.43 & 0.13 & 0.36 & 2.59 & 0.40 \\
\hline $0456.2+1554$ & K7 & 12.77 & 0.73 & 0.74 & -0.03 & 2.61 & 0.00 \\
\hline $0457.0+1600$ & M1 & 14.42 & 0.89 & 0.68 & 0.00 & 2.52 & 0.23 \\
\hline HD 286179 & G3 & 10.34 & 0.45 & 0.15 & 0.31 & 2.61 & 0.42 \\
\hline $0457.5+2014$ & K3 & 11.11 & 0.53 & 0.36 & 0.26 & 2.61 & 0.00 \\
\hline $0458.7+2046$ & K7 & 11.90 & 0.72 & 0.84 & 0.06 & 2.55 & 0.00 \\
\hline
\end{tabular}

Notes to Table:

$\dagger$ classified as WTTS by Wichmann (1994).

$\ddagger 04253+2618$ SpT. M0, $W(\mathrm{H} \alpha)=-2.26 \AA,=\mathrm{J} 4872$.

on the figure the mean ZAMS, giant and sub-giant sequences, adapted from Olsen's $(1983,1984)$ data for stars with spectral types later than $\approx$ G2. In Fig. 8 the lower envelope of the ZAMS (thin solid line), giant and subgiant $\left(\beta,\left[m_{1}\right]\right)$ sequences (broken line and thick solid line respectively) are also indicated. We adopted the mean IS reddening law given by Mathis (1990) to obtain the coefficients that define the (reddening-free) colour indices $\left[m_{1}\right]$ and $\left[c_{1}\right]$. For the definition of the indices, see Strömgren 


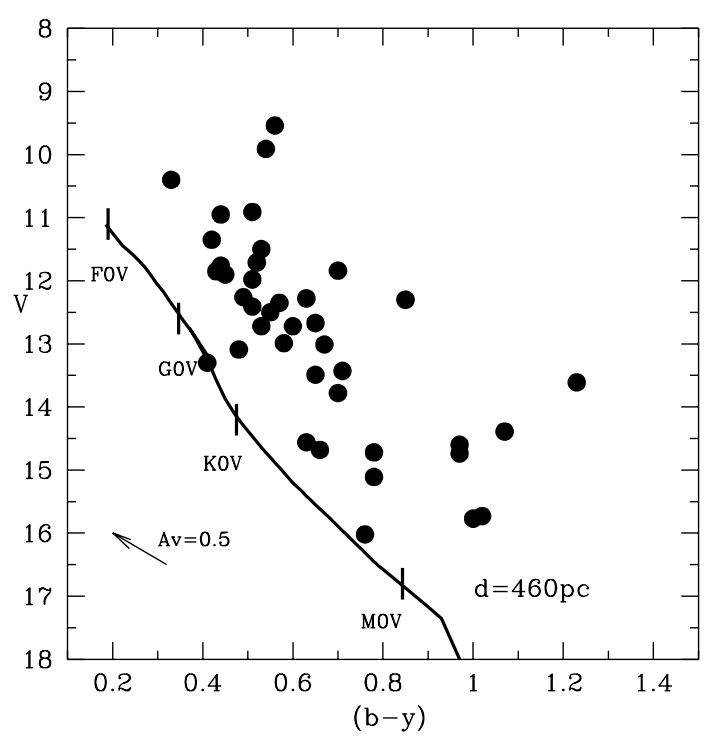

Fig. 4. Colour-magnitude diagram of the WTTS in Orion. The ZAMS scaled to the distance of $460 \mathrm{pc}$ is indicated in the figure by the solid line. The direction of the normal reddening vector is also shown in the figure

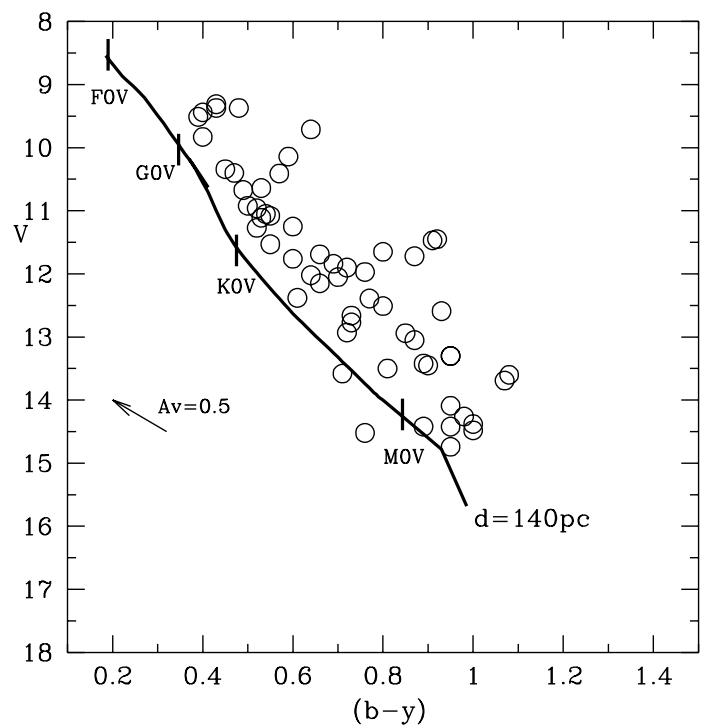

Fig. 5. Colour-magnitude diagram of the WTTS in TaurusAuriga. The position of the ZAMS scaled to the distance of $140 \mathrm{pc}$ is depicted with the solid line

1966 or T94. For the sake of simplicity, in Fig. 8 we plotted only the program stars with spectral types earlier than K7. For later spectral types the reference lines turn to the left in the diagram because of the behaviour of the $\left[m_{1}\right]$ index (cf. Fig. 7).

From a quick inspection of Figs. 7 and 8 it is apparent that, for the later spectral types (G2 or later), the $\left(\left[m_{1}\right],\left[c_{1}\right]\right)$ and $\beta,\left[m_{1}\right]$ diagrams are sensitive to the stellar temperature through the $\left[m_{1}\right]$ index and luminosity- or gravity-dependent through the $\left[c_{1}\right]$ and $\beta$ indices. Because of this, the $\left(\left[m_{1}\right],\left[c_{1}\right]\right)$ diagram is an observational HRdiagram but with the advantage that it is distance- and reddening-independent. One further infers that a large

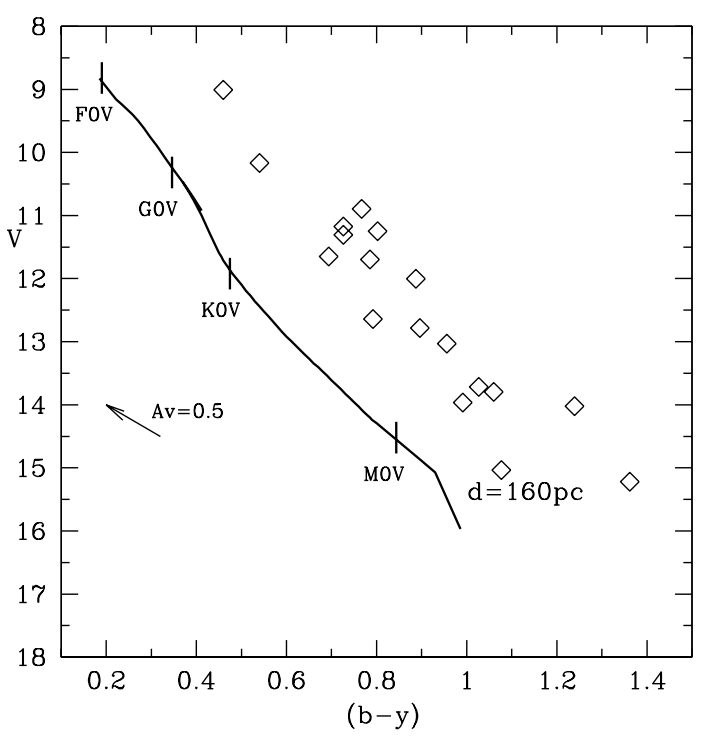

Fig. 6. Colour-magnitude diagram of the WTTS in Scorpius OB2-2 and Ophiuchus. The position of the ZAMS scaled to the distance of $160 \mathrm{pc}$ is shown with the solid line

fraction of the program stars are colder and less gravitative than their dwarf counterparts, with an upper limit for the temperature $T_{\text {eff }} \leq 6500 \mathrm{~K}$ (spectral type $\mathrm{F} 6$ or later) and hence lie in the expected domain for young stars with enhanced LiI $\lambda 6708 \AA$ absorption. Five stars seem to have gravities suitable of giant stars (cf. Fig. 7). A large fraction of the sample stars typically have luminosity class between III and V but the diagrams show a large scatter, with many stars out of bounds. This was due to i) the fact that, originally we wanted only to obtain the magnitude $V$ and the color $(b-y)$ of the program stars, in order to derredden the data and fix the stars in a luminosity-temperature diagram using bolometric corrections and ii) that some program stars still suffer of veiling, making their $[c 1]$ and $[m 1]$ indices bluer than expected for their spectral class. We also make the remark that the $c_{\circ}$ and $m_{\circ}$ color indices were obtained as a byproduct since the photometer measures all four filter bands simultaneously. The composed colours should be measured more accurately to be conclusive, particularly for late type stars. In any case, one should be aware that an error of 0.2 in the $\left[m_{1}\right]$ index causes a classification error of two spectral subclasses, but the same error in $\left[c_{1}\right]$ leads to a difference between a giant and a dwarf star. Anyhow, this result for the sample reassures us that many program stars are PMS objects. We also note that the sample in Taurus-Auriga is hotter than the sample in Orion.

From Fig. 8 it is also apparent that some stars scatter all over the diagram, mimicking gravitative objects, stars with strong winds or stars with $\mathrm{H} \beta$ filled-in with emission or in emission. The program stars with $\mathrm{H} \alpha$ in emission from the literature, are indicated with crosses in the figure. We conclude that little or no additional information can be drawn from the location of the program stars in Fig. 8. 
Table 4. uvby- $\beta$ photometry of WTTS in Scorpius OB2-2 and in Ophiuchus

\begin{tabular}{llrlrrrr}
\hline Name/RXJ & Sp.T. & $V$ & $b-y$ & $m 1$ & $c 1$ & $\beta$ & $A_{V}$ \\
\hline & & & & & & & \\
$155203-2338$ & G2IV & 9.01 & 0.46 & 0.19 & 0.30 & 2.56 & 0.48 \\
$155331-2340$ & M1.5 & 13.03 & 0.96 & 0.47 & 0.18 & 2.64 & 0.53 \\
$155421-2330$ & M0 & 12.78 & 0.90 & 0.69 & -0.24 & 2.54 & 0.48 \\
$155427-2346$ & M0.5 & 13.80 & 1.06 & 0.36 & 0.18 & 2.59 & 1.27 \\
$155703-2212$ & M1 & 13.72 & 1.03 & 0.48 & 0.05 & 2.61 & 0.99 \\
$155828-2232$ & K1IV & 11.65 & 0.69 & 0.37 & 0.23 & 2.57 & 1.07 \\
$155913-2233$ & K5IV & 11.31 & 0.73 & 0.46 & 0.14 & 2.59 & 0.34 \\
$160153-1922$ & K2IV & 11.17 & 0.73 & 0.37 & 0.23 & 2.58 & 1.09 \\
$160233-1931$ & M1 & 13.96 & 0.99 & 0.51 & -0.04 & 2.66 & 0.79 \\
$160728-1856$ & M1 & 15.03 & 1.08 & 0.29 & 0.36 & 2.75 & 1.27 \\
$161431-2256$ & G0IV & 10.17 & 0.54 & 0.15 & 0.34 & 2.54 & 1.08 \\
$162649-2145$ & K0IV & 11.25 & 0.80 & 0.30 & 0.29 & 2.56 & 1.83 \\
Oph1 & K2 & 12.00 & 0.89 & 0.30 & 0.27 & 2.60 & 1.99 \\
Oph2 & K1 & 11.70 & 0.79 & 0.28 & 0.25 & 2.58 & 1.58 \\
Oph3 & K0 & 10.89 & 0.77 & 0.34 & 0.22 & 2.59 & 1.63 \\
Oph4 & K4 & 14.0 & 1.24 & 0.4 & 0.43 & 2.41 & 3.52 \\
Oph5 $\dagger$ & M2 & 15.22 & 1.36 & 0.35 & 0.70 & 2.87 & 0.15 \\
Oph6 $\dagger$ & K7 & 12.64 & 0.79 & -0.12 & $-1.5:$ & 2.75 & 2.74 \\
& & & & & & & \\
\hline
\end{tabular}

Notes to table: ${ }^{\dagger}$ CTTS, after Wa88.

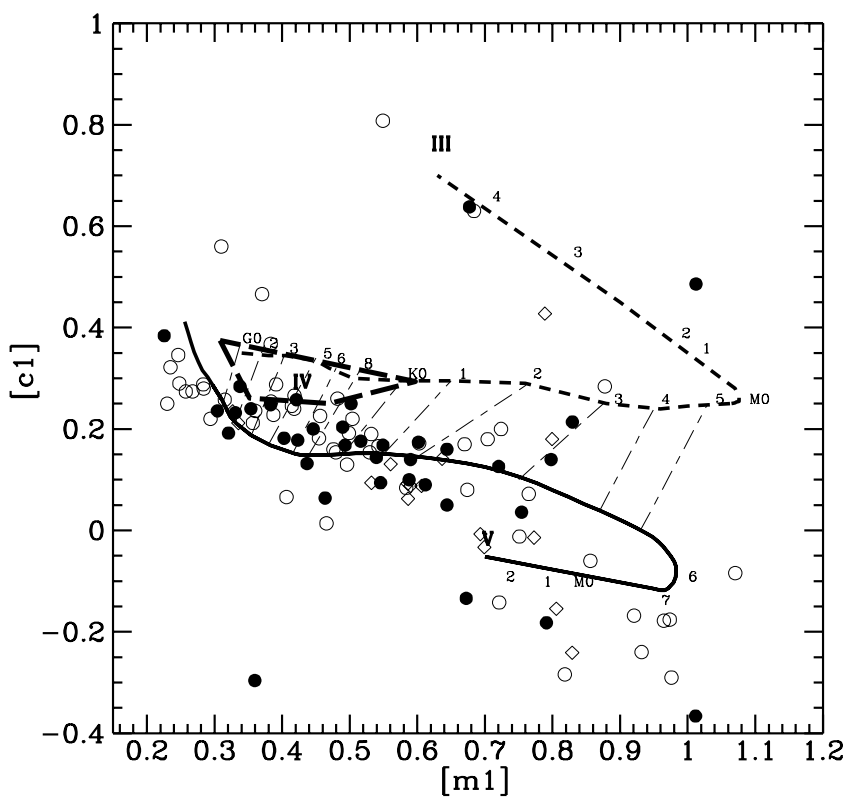

Fig. 7. The ([m $\left.\left.m_{1}\right],\left[c_{1}\right]\right)$ diagram of the WTTS in the SFRs studied: Orion (filled circle), Taurus-Auriga (open circle), Scorpius OB2-2 (diamond). The thick line represents the ZAMS

From the spectral types of the program stars given in the literature and the photometric indices given here we also conclude that the $\left[m_{1}\right]$ colour index enables us to give a good stellar temperature estimate $T_{*}$ for the program stars (typically within a subclass). We also find that the program stars are for the most part about half a spectral subclass colder than their main-sequence counterparts.

In Fig. 9 we show the equivalent width $W(\mathrm{H} \alpha)$ as a function of the $\beta$ index for the program stars. The $\beta$ data were taken from Tables 2, 3 and 4 of this work and the $W(\mathrm{H} \alpha)$ data were taken from A96, Wi96 and Wa94. Also shown in the figure is the expected relation between $\beta$ and

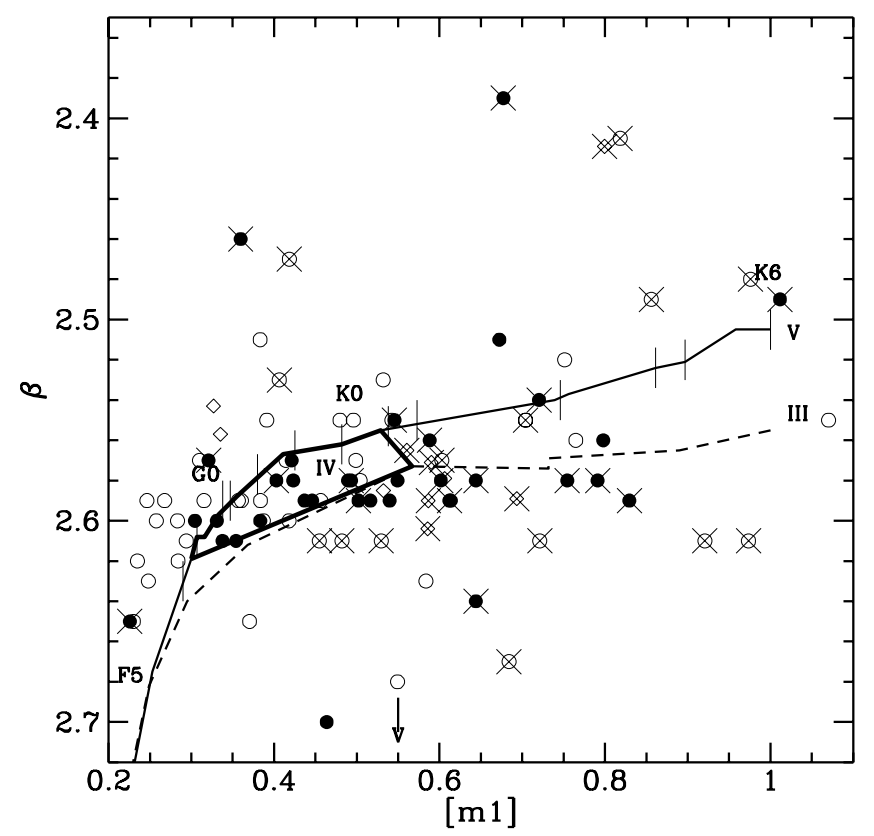

Fig. 8. The $\left(\beta,\left[m_{1}\right]\right)$-diagram of the WTTS. Symbols as Fig. 7. For clarity, only the WTTS earlier than K7 are plotted in the figure. The program stars with $\mathrm{H} \alpha$ in emission are indicated with crosses

$W(\mathrm{H} \alpha)$ for dwarfs (dotted line) and giants (solid line). The $W(\mathrm{H} \alpha)$ values for the reference lines were obtained by measuring the equivalent width of the $\mathrm{H} \alpha$ line for a suitable sample of dwarf and giant stars selected from the library of spectra by Montes et al. (1997), while the $\beta$ values were obtained from Hauck \& Mermilliod (1998). From the figure we notice that, besides the fact that the $\beta$ and $W(\mathrm{H} \alpha)$ values were not determined simultaneously for stars with $\mathrm{H} \alpha$ in emission, the $\beta$ index is usually also indicative of emission. From Fig. 9 we also conclude that most of our sample stars have $\mathrm{H} \alpha$ filled-in or in emission.

Sixteen of the eighteen WTTS with a photometric rotational period studied by Bouvier et al. (1997) are contained in our sample of program stars in Taurus. In general, their visual magnitudes coincide with our values within the observational errors and the intrinsic variability of the objects in common $(\delta V$ (ours - bou97) $=+0.049$, $\left.\sigma_{\delta V}=0.091\right)$, except for RXJ0420.3+3123: this star deviates by more than $\delta V=-0.57$. Its range of variability is found to be only 0. 07 (cf. Bouvier et al. 1997). Excluding this star, we expect to obtain the same physical parameters for the subsample of stars in common with Bouvier et al. (1997), such as $A_{V}, T_{\text {eff }}, \log \left(L_{*} / L_{\odot}\right)$, $\tau_{\text {age }}$, etc., if we assume that they all belong to the Taurus-Auriga SFR. We find a similar situation for the WTTS associated with the Scorpius OB2-2 association, since our data matches that of Wa86 and Wa94, except for two of the objects: Oph6 and RXJ155703-2212. Oph6 is a $C T T S$ and its range of variations can be larger than that quoted by Wa94; RXJ155703-2212 is almost 0.5 


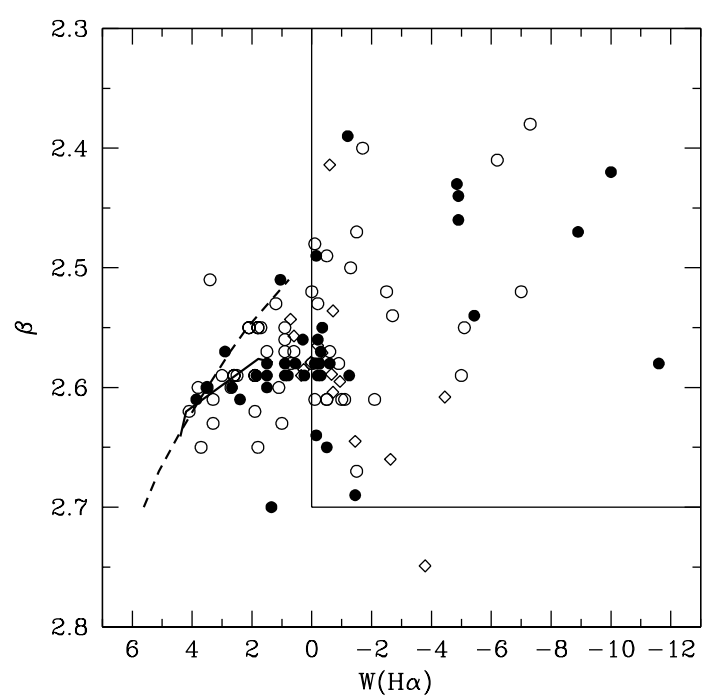

Fig. 9. The $\beta$ vs. $W(\mathrm{H} \alpha)$-diagram of the WTTS. The dotted and the solid lines represent the expected $\beta$ vs. $W\left(\mathrm{H}_{\alpha}\right)$ relations for dwarf and giant stars respectively. The filled and open circles represent the sample stars in Orion and Taurus-Auriga, respectively. The diamonds represent objects in Scorpius OB22 and Ophiuchus. For more details, consult Sect. 3.2.2

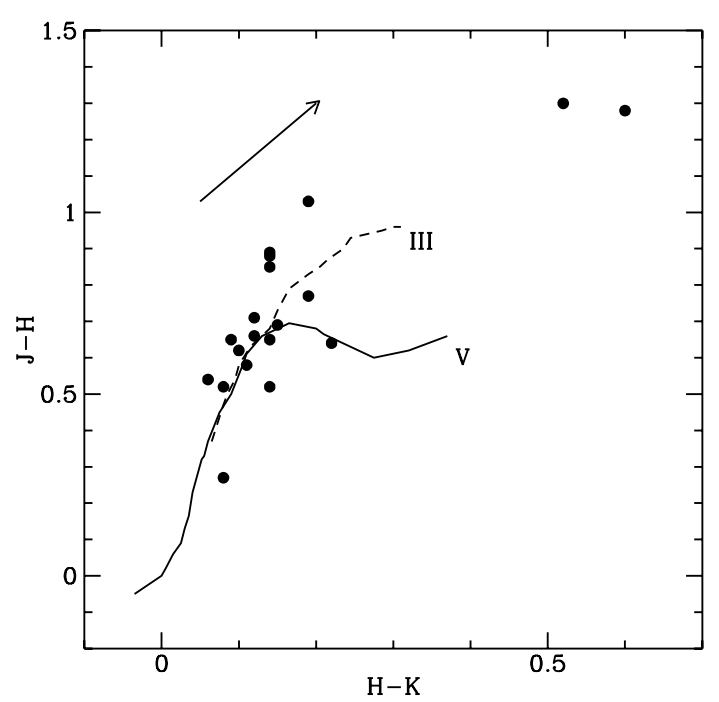

Fig. 10. The $(J-H)$ vs. $(H-K)$ diagram for the WTTS in Taurus-Auriga. The solid line is the location of the main sequence and the broken line the giant sequence, adapted from the data given by Bessell \& Brett (1988). The arrow indicates the direction of the (normal) reddening vector

fainter now than its brightness given by Wa94 earlier. We find no clear explanation for this change.

However, as mentioned before in Sects. 3.1 and 3.2, some of the program stars can be foreground objects and a better distance estimate for these stars is required in order to derive their physical parameters. The uvby- $\beta$ photometry given here provides such a possibility (Terranegra \& Chavarria-K 1999).
Figure 10 shows the location in the two-colour $(J-H$, $H-K$ ) diagram of the WTTS in Taurus-Auriga. Also given in the figure are the loci of the main (solid line) and giant (broken line) sequences, taken from Bessel \& Brett (1988). One readily sees from Fig. 10 that, except for two stars which are highly reddened with an apparently normal IS extinction law, namely the CTTS RXJ0433.9+2613 (IT Tau) and RXJ0425.3+2618 (J4872), the rest of the WTTS have, within the observational errors, similar IR colors to those of normal field dwarf stars later than K2. A more detailed examination of the program stars with optical and IR photometric data indicates that, on average, the WTTS have a slight near IR excess, probably due to a remnant of circumstellar matter. Finally, integrating under the dereddened (broad) spectral flux distribution derived from the near IR and optical data of 19 stars in Taurus-Auriga in order to calculate their bolometric luminosity, we confirm Wa94's result that the stars follow, within the observational errors $\left(\sigma\left(L_{*}\right) \leq 0.1 \mathrm{dex}\right)$, the bolometric correction relations for dwarfs and giants (e.g. Schmidt-Kaler 1982). Thus, luminosities derived from the dereddened visual magnitude and colour using bolometric corrections are good estimates for our purposes.

In conclusion, in this work we show, independently of any distance estimate, that, in general, the program stars are more luminous than ZAMS stars, typically with luminosity classes between III and V (cf. the $\left(\left[m_{1}\right],\left[c_{1}\right]\right)$ and $\left(\beta,\left[m_{1}\right]\right)$ diagrams), giving support to the premise that they are young (PMS) objects and in agreement with their activity indicators provided by the spectroscopy. Another important result is that the WTTS and WTTS candidates are a mixture of objects belonging to the SFRs studied here and of foreground and yet young (i.e. PMS) stars, since they still do not reach the main sequence. Some objects are too far from the assumed parent SFR to be explained by isotropic drifting or slingshot mechanisms (cf. Herbig 1978; Sterzik \& Durisen 1995, respectively). Some of the stars could belong to the Gould Belt population or, less probably, they were formed locally (cf. Guillout et al. 1998; Feigelson 1996, respectively).

Much observational work still has to be done in order to elucidate their true nature and $u v b y-\beta$ is one of the techniques that enables us to answer some of these matters.

Acknowledgements. We thank Juan Manuel Alcalá and Rainer Wichmann for making many program stars in Orion and Taurus-Auriga regions available to us prior to their publication. We appreciate the useful comments and suggestions by the referee, R. Wichmann, Juan Manuel Alcalá and Michael Sterzick for interesting and fruitful discussions about WTTS, and the technical and administrative staff of SPMO for their continuous and enthusiastic support in the realization of the observing runs. C. Chavarría-K thanks Prof. M. Capaccioli for making his stay at the OAC-Napoli possible. Christine Harris proofread the manuscript. 
This work was partially supported by Consejo Nacional de Ciencia y Tecnología, México (projects 400340-4-2243 PE and 400354-5-27757 E), and by the CNR-GNA98 and COFIN98MURST, Italy.

\section{References}

Alcalá J.M., Krautter J., Schmitt J.H.M.M., Covino E., Wichmann R., Mundt R., 1995, A\&AS 114, 109

Alcalá J.M., Terranegra L., Wichmann R., et al., 1996, A\&AS 119, 7 (A96)

Alcalá J.M., Terranegra L., Chavarría-K C., 1998, A\&A 330, 1017 (A98)

Bessell M.S., Brett J.M., 1988, PASP 100, 1134

Bodenheimer P., 1965, ApJ 142, 459

Bouvier J., Wichmann R., Grankin K., et al., 1997, A\&A 318, 495

Carrasco L., Recillas-Cruz E., García-Barreto A., et al., 1991, PASP 103, 987

Chavarría A., de Lara E., Chavarría-K C., 2000, ArcoIris.v02 (RainBow.v02), a multiwavelength reduction program for IBM compatible PC's. Instituto de Astronomía, U.N.A.M., México

Chavarría-K C., Moreno-Corral M.A., de Lara E., 1995, Rev. Mex. Astron. Astrofis. (Suppl.) 3, 117

Cohen M., Kuhi L.V., 1979, ApJS 41, 743

Crawford D.L., 1979, AJ 84, 1858

Crawford D.L., Barnes J.V., Golson J.C., 1971, AJ 76, 1058

Crawford D.L., Barnes J.V., Golson J.C., Hube D.P., 1973, AJ 78,738

Feigelson E.D., 1996, ApJ 468, 306

Guillout P., et al., 1998, A\&A 337, 113

Hauck B., Mermilliod M., 1998, A\&AS 129, 431

Herbig G.H., 1978, in Problems of physics and evolution of the Universe, Miroyan L. (ed.). Academy of Science of Armenia, Erevan, p. 171

Herbig G.H., Bell K.R., 1988, Lick Obs. Bull. No. 1111

Krautter J., Wichmann R., Schmitt J.H.M.M., et al., 1997, A\&AS 123, 329

Li J.Z., Hu J.Y., 1998, A\&AS 132, 173
Maggazù A., Martín E.L., Sterzik M.F., Neuhäuser R., Covino E., Alcalá J.M., 1997, A\&AS 124, 449

Mathis J.S., 1990, ARA\&A 28, 37

Mitchell R., 1960, ApJ 132, 68

Montes D., Martín E.L., Fernández-Figueroa M.J., Cornide M., De Castro E., 1997, A\&AS 123, 473

Montmerle Th., Feigelson F.D., Bouvier J., André P., 1993, Protostars and Planets III, Levy E., Lumine J. (eds.). University of Arizona Press, p. 689

Nissen P.E., 1984, User's manual of the Danish uvby- $\beta$ photometer. Instituto de Astronomía, México

Olsen E.H., 1983, A\&AS 54, 55

Olsen E.H., 1984, A\&AS 57, 443

Sciortino S., Damiani F., Favata F., Micela G., 1998, A\&A 332, 825

Schmidt-Kaler T.H., 1982, in Physical parameters of stars, Landolt-Börnstein New Series, Vol. 2b, Astronomy and Astrophysics, Stars and Clusters, Schaifers K., Voigt H.H. (eds.). Springer Verlag, New York

Shu F.H., Adams F.C., Lizano S., 1987, ARA\&A 25, 23

Sterzik M.F., Durisen R.H., 1995, A\&A 304, L9

Strömgren B., 1966, ARA\&A 4, 433

Tapia M., Neri L., Roth M., 1986, Rev. Mex. Astron. Astrofis. 13,115

Terranegra L., Chavarría-K C., Diaz S., González-Patiño D., 1994, A\&AS 104, 557 (T94)

Terranegra L., Chavarría-K C., 1999, in "Second Three-Islands Euroconference on Stellar Clusters and Associations: Convection, Rotation and Dynamos", Mondello, Palermo, Italy

Walter F.M., 1986, ApJ 306, 573 (Wa86)

Walter F.M., Brown A., Mathieu R.D., Myers P.C., Vrba F.J., 1988, AJ 96, 297 (Wa88)

Walter F.M., Vrba F.J., Mathieu R.D., Brown A., Myers P.C., 1994, AJ 107, 692 (Wa94)

Wichmann R., 1994, Ph.D. Dissertation, Heidelbeg, Germany Wichmann R., Krautter J., Schmitt J.H.M.M., et al., 1996, A\&A 312, 439 (Wi96)

Wichmann R., Krautter J., Covino E., Alcalá J.M., Neuhäuser R., Schmitt J.H.M.N., 1997, A\&A 320, 185 